\title{
A VARIANT OF A FIXED POINT THEOREM OF BROWDER-FAN AND REICH
}

\author{
V. M. SEHGAL \\ Department of Mathematics \\ University of Wyoming \\ Laramie, Wyoming 82071 U.S.A. \\ (Received January 8, 1985)
}

ABSTRACT. Let $S$ be a convex, weakly compact subset of a locally convex Hausdorff space $(E, T)$ and $f: S \rightarrow E$ be a continuous multifunction from its weak topology $\omega$ to $\tau$. Let $P$ be a continuous seminorm on $(E, \tau)$ and for subsets $A, B$, of $E$, let $p(A, B)=\inf : p(x-y): x \in A, y \in B\}$. In this paper, sufficient conditions are developed for the existence of an $x \in S$ satisfying $p(x, f x)=p(f x, S)$. The result is then used to prove several fixed point theorems.

KEY WORDS AND PHAASAS. Multifunctions, convex topology, fixed point:.

1:1:0 AMS SIJBJECT CLASSIFICATION CODES. 47H10, 60H25.

\section{INTRODUCTION}

Let $(E, \tau)$ be a locally convex Hausdorff topological vector space with topology $\tau$ and $E^{*}=(E, \tau)^{*}$ be its topological dual. Let $\omega=\omega\left(E, E^{*}\right)$ be the weak topology of $E$. Let $P$ and $Q$ denote the family of continuous semi-norms generating the topologies $\tau$ and $\omega$ respectively. For sets $A$ and $B$ of $E$ and $a \in P$, let $p(A, B)=\inf \{p(x-y): x \in A, y \in B\}$. In this paper, we prove the following result.

THEOREM 1 . Let $S$ be a nonempty convex, $\omega$-compact subset of $E$ and $f:(S, \omega)$ $\rightarrow(E, r)$ be a continuous multifunction such that $f(x)$ is convex and w-compact for each $x \in S$. Then for each $p \in P$ there exists a $x \in S$ satisfying

$$
p(x, f x)=p(f x, S) \text {. }
$$

Futher if $p(x, f x)>0$ then $x \in \partial(S, i)) n \partial(S, \tau)$ where $\partial$ denotes the boundary.

It may be remarked the since $A \subseteq r$. $f$ in Theorem $l$ is also a continuous multifunction from $\left(S, A_{1}\right) \rightarrow\left(E,{ }^{\prime}\right)$. Consequently it follows by Reich (Lemma 1.6 [1]) that each $q \in Q$ satisfies (1.1) for some $x \in S$. However, since $Q \subseteq P$, the lemma in [1] is not applicable for arbitrary $p \in P$. In fact, Theorem 1 contains the above lemma [1] (see Corollary 2) and it provides a generalization of a well-known result of Ky Fan [2] for single valued mappings.

2. PRELIMINARY RESULTS.

Recall that if $X, Y$ are topological spaces then a multifunction $f: X \rightarrow Y$ ( $f x \neq \emptyset$ for each $x$ ) is upper (lower) semicontinuous iff for each closed (open) sub- 
set $\Lambda$ of $Y, f^{-1}(A)=\{x \in X: f(x) \cap A \neq \emptyset\}$ is a closed (open) subset of $X$. It follows by definition that $f$ is 1.s.c. iff fx $\cap U \neq \emptyset$ for some open set $U$ of $Y$ and $x$ in $X$ then $f z \cap U \neq \emptyset$ for each $z$ in some neighborhood $V$ of $X$. Further, it is well-known (i) that if $f$ is u.s.c. and a net $x_{\alpha}+x$ in $x$ and $y_{\alpha} \rightarrow y$ in $Y$ with $y_{\alpha}=f_{x}$ then $y=f x$; (ii) if $x$ is compact and $f$ is u.s.c. with compact values then $\mathrm{fX}$ is compact. A multifunction which is both u.s.c. and 1.s.c. is called continuous.

We prove two lemmas that simplify the proof of Theorem 1 . Throughout, let $E$ be as stated in the beginning and $S$ a nonempty subset of $E$.

LEMPI 1. Let $A, B$ be $(1$-compact sets of $E$ and $p \in P$. Then $p(A, B)=p(x, B)$ $=p(x-y)$ for some $x \in A, y \in B$.

PROOF. Chonse sequences $\left\{x_{n}\right\} \subseteq A,\left\{y_{n}\right\} \subseteq B$ such that $p\left(x_{n}-y_{n}\right) \downarrow p(A, B)$. We may assume that $x_{n} \rightarrow x$ weakly for some $x \in A$ and $y_{n} \rightarrow y$ weakly for some $y \in B$. By Hahn Banach Theorem (see [3], Cor. 2, p. 29) there exists a $x^{*}=E^{*}$ with $x^{*}(x-y)$ $=p(x-y)$ and $\left|x^{*}(u)\right| \leq p(u)$ for each $u \in E$. Consequently, since $x_{n}-y_{n} \rightarrow$ $\mathrm{x}-\mathrm{y}$ weakly, $p(x, B) \leq p(x-y)=x^{*}(x-y)=\lim \left|x^{*}\left(x_{n}-y_{n}\right)\right| \leq \underline{1 i m} p\left(x_{n}-y_{n}\right)=p(\Lambda, B) \leq p(x, B)$

LEMM 2. Let $S$ be $\omega$-compact subset of $E$ and $f:(S, \omega) \rightarrow(E, \tau)$ be a 1.s.c. multifunction with weakly compact values. If a net $x_{\alpha} \rightarrow x$ weakly in $S$, then for each $p \in P$ and $r>0, p\left(f x_{\alpha}, S\right) \leq p(f x, S)+\varepsilon$ eventually.

PROOF. It follows by Lemma $l$ that there is a $y \in f x$ with $p(f x, S)=p(y, S)$. Let $U=\{x \in E: p(x-y)<\varepsilon\}$. Then $U$ is $\tau$-open and $y \in f x \cap U$. llence by 1.s.c., $f x_{\alpha} \cap U \neq \emptyset$ eventually. For such $\alpha$, let $y_{\alpha}=f x_{\alpha} \cap U$. Then eventually,

$$
p\left(f x_{\alpha}, S\right) \leq p\left(y_{\alpha}, S\right) \leq p\left(y_{\alpha}-y\right)+p(y, s) \leq p(f x, S)+\varepsilon .
$$

3. MAIN RESULTS.

PROOF OF THEOREM 1. Let $p \in P$. Define a multifunction $g:(S, \omega) \rightarrow(S, \omega)$ by $g(x)=\{y \in S: p(y, f x)=p(f x, s)\}$.

Then by Lemma $1, \mathrm{~g}(\mathrm{x}) \neq \emptyset$ and is clearly convex. Further, since $\mathrm{S}$ is $\tau$-closed and for any $y, z \in g(x)$, the triangular inequality implies

$$
|p(y, f x)-p(z, f x)| \leq p(y-z) \text {. }
$$

It follows $\mathrm{g}(\mathrm{x})$ is $\tau$-closed convex and hence a $\omega$-compact subset of $\mathrm{S}$. We show that $g$ is u.s.c. Let $C$ be a weakly closed (hence weakly compact) aubset of $S$. We show that $x \in g^{-1}(C)$, that is $g(x) \cap C \neq \emptyset$. Choose for each $\alpha, y_{\alpha} \in g x_{\alpha} \cap C$. We may assume that $y_{\alpha} \rightarrow y$ weakly for some $y \in C$. Also since $p\left(y_{\alpha}, f x_{\alpha}\right)=$ $\mathrm{p}\left(\mathrm{fx_{ \alpha }}, \mathrm{S}\right)$, there exists $z_{\alpha}=f x_{\alpha}$ with $\mathrm{p}\left(\mathrm{y}_{\alpha}-\mathrm{z}_{\alpha}\right)=\mathrm{p}\left(\mathrm{fx_{ \alpha }}, \mathrm{S}\right)$. Further $\mathrm{f:}(\mathrm{S}, \omega)$ $\rightarrow(E, w)$ being u.s.c., it follows that $f S$ is weakly compact and hence we may assume that $z_{\alpha} \rightarrow z$ weakly for some $z \in f x$. Thus $y_{\alpha}-z_{\alpha} \rightarrow y-z$ weakly. Choose as before $a x^{*} \in E^{*}$ such that $x^{*}(y-z)=p(y-z)$ and $\left|x^{*}(u)\right| \leq p(u)$ for each u E. Let $\varepsilon>0$. Choose $\alpha_{0} \in \Delta$ such that $\mathrm{p}\left(\mathrm{fx} \mathrm{x}_{\alpha}, \mathrm{S}\right) \leq \mathrm{p}(\mathrm{fx}, \mathrm{S})+\varepsilon$ for $\alpha \geq \alpha_{0}$. Consequently, for $\alpha: \alpha_{0}\left|x^{*}\left(y_{\alpha}-z_{\alpha}\right)\right| \leq p\left(f_{\alpha}, s\right) \leq p(f x, s)+\varepsilon$ and hence

$$
p(y, f x) \leq p(y-z)=\lim \left|x^{*}\left(y_{\alpha}-z_{\alpha}\right)\right| \leq p(f x, S)+\varepsilon .
$$


Since $\varepsilon>0$ is arbitrary and $p(f x, S) \leq p(y, f x)$, we have $p(y, f x)=p(f x, S)$ that is $y \in g(x) \cap C$. Thus $g$ is u.s.c. Hence by Glicksberg [4] there exists a $x \in S$ with $x \in g(x)$. This implies $p(x, f x)=p(f x, s)$.

Now, suppose $p(x, f x)>0$. Then $f x \cap S=\emptyset$. Choose by Lemma 1 , a $y \in f x$ satisfying $p(x-y)=p(x, f x)$. Now, if $x \in \operatorname{int}(S, \omega)$ or int $(S, \tau)$, then since $S$ being weakly closed and convex, there is a $z \in(x, y) \cap S$ with $0<p(f x, S) \leq$ $p(y-z)<p(x-y)=p(f x, S)$, a contradiction. This proves the result.

As a consequence of Theorem 1, we have

COROLLARY 1. Let $S$ be a convex and weakly compact set in $E$ and $f:(S, \omega) \rightarrow$ $(E, \tau)$ be a continuous multifunction with convex and $\omega$-compact values. Then either $f$ has a fixed point or there exists a $P \in P$ and $x \in S$ satisfying $0<p(x, f x)=$ $\mathrm{p}(\mathrm{Ex}, \mathrm{S})$.

PROOF. For each $p \in P$, let $x_{p} \in S$ satisfying (1). If $p\left(x_{p}, f x_{p}\right)=0$ for each $p \in P$, then using the implication that $f:(S, \omega) \rightarrow(S, \omega)$ is continuous, it follows that $A_{p}=\{x \in S: p(x, f x)=0\}$ is nonempty, weakly compact and the family $\left\{A_{p}: p \in P\right\}$ has finite intersection property. Consequently, there exists $x \in S$ with $p(x, f x)=0$ for each $p \in P$. Now, if $x \notin f x$, then since $x-f x$ is $\tau-c$ losed and convex and $0 \notin x-f x$, there exists (see [3], Cor. 1, p. 30) a $x^{*} \in E^{*}$ such that $0 \notin\left\{x^{*}(x-y): y \in f x\right\}$. Let $p=\left|x^{*}\right|$. Then $p \in P$ and $p(x, f x) \neq 0$, a contradiction.

The following corollaries result from Theorem 1.

COROLLARY 2. (Reich $[1]$ ). Let $S$ be a compact and convex in $(E, \tau$ ) and $f$ : $(S, \tau) \rightarrow(E, \tau)$ be a continuous mutifunction with convex and compact values. Then either $f$ has a fixed point or there exists a $p \in P$ and $x \in S$ satisfying $0<$ $p(x, f x)=p(f x, S)$.

COROLLARY 3. (Waters [5]). Let $S$ be a compact and convex subset of (E, $\tau$ ) and $\mathrm{f}:(\mathrm{S}, \tau) \rightarrow(E, \tau)$ be a continuous multifunction with convex and weakly compact values. Then for each $P \in P$, there exists $a x \in S$ satisfying (1.1).

PROOF. It suffices to show that the hypotheses in Corollary 2 and Corollary 3 imply that $\mathrm{f}:(\mathrm{S}, \omega) \rightarrow(\mathrm{E}, \tau)$ is a continuous multifunction. Let $\mathrm{A}$ be $\tau$-closed in $E$. Then $f^{-1}(A)$ is $\tau$-compact subset of $S$. Since $S$ is weakly closed, it follows that $f^{-1}(A)$ is weakly closed. Thus $f$ is u.s.c. Similarly if $A$ is $\tau$-open set in $E$ then $S \backslash f^{-1}(A)=f^{-1}(E \backslash A)$ is $\omega$-closed and hence $f^{-1}(A)$ is $w$-open. Thus $\mathrm{f}$ is $1 . \mathrm{s.c}$.

In the setting of semi-reflexive locally convex spaces, we have

COROLLARY 4. Let $S$ be a closed, bounded and convex subset of a semi-reflexive locally convex space $E$. If $f:(S, \omega) \rightarrow(E, \tau)$ is continuous multifunction with closed, bounded and convex values then for each $p \in P$, there exists $x \in S$ satisfying (1.1).

*Theorem 1 of this paper was presented at the summer meeting of the Amer. Math. Society, (1983), Albany, New York. 


\section{REFERENCES}

1. REICH, S. Fixed point theorems in locally convex spaces, Math. Z. 125 (1972), pp. 17-31.

2. KY FAN Extensions of two fixed point theorems of F. E. Browder, Math. Z. 112 (1969), pp. 234-240.

3. ROBERTSON, A. P., and W. J. Topological Vector Spaces, Cambridge, University Press (1966).

4. GLICKSBERG, I. L. A further generalization of Kakutani's fixed point theorem with application to Nash equilibrium points, Proc. Amer. Math. Soc. 3 (1952), pp. 170-174.

5. WATERS, C. Ph.D. Thesis (1984), University of Wyoming. 


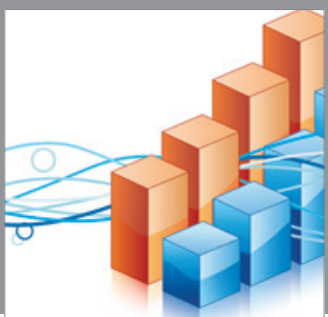

Advances in

Operations Research

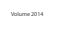

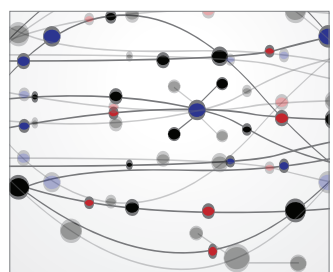

\section{The Scientific} World Journal
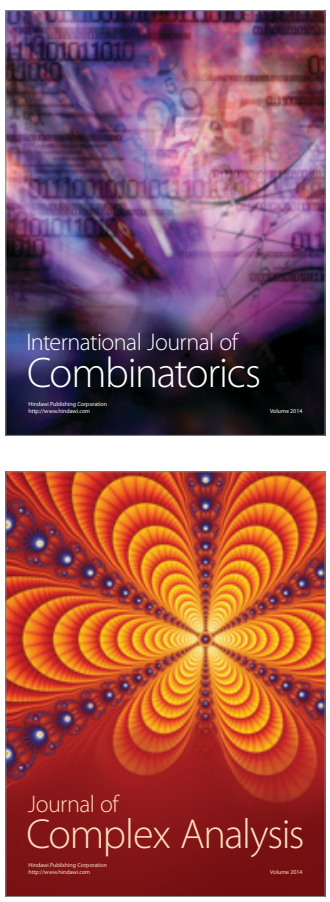

International Journal of

Mathematics and

Mathematical

Sciences
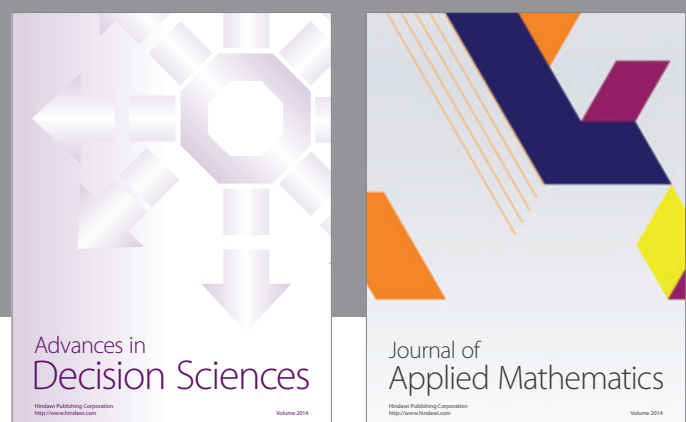

Journal of

Applied Mathematics
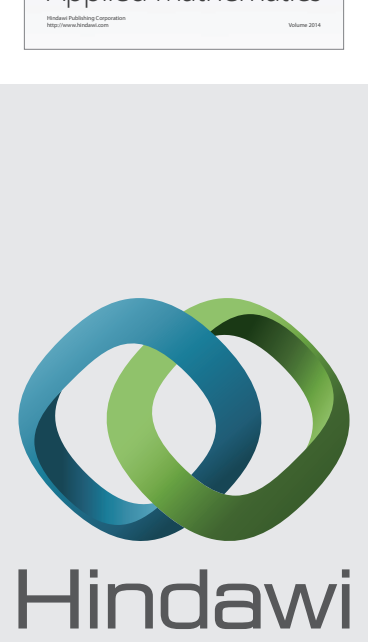

Submit your manuscripts at http://www.hindawi.com
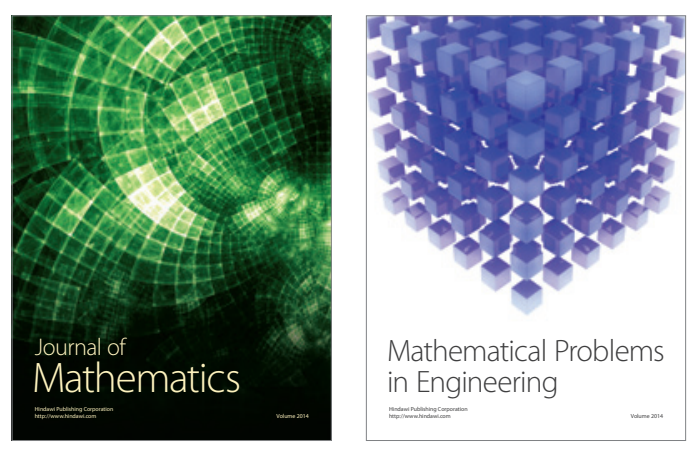

Mathematical Problems in Engineering
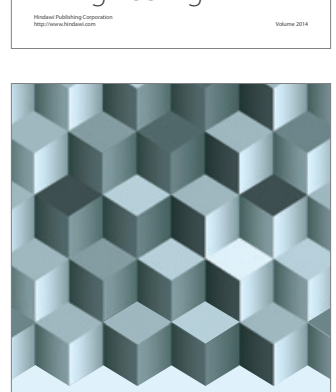

Journal of

Function Spaces
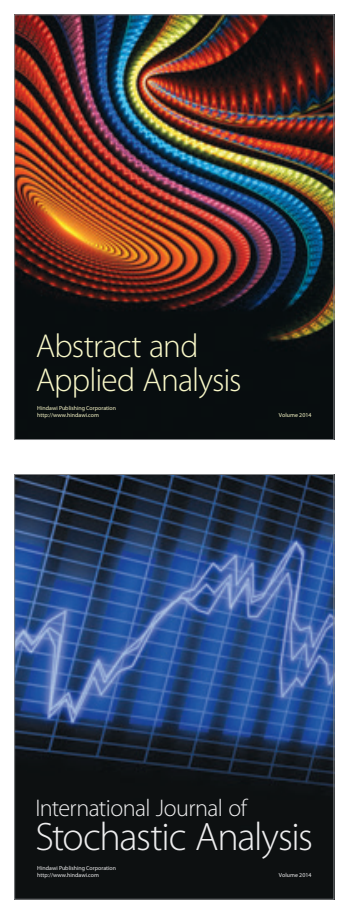

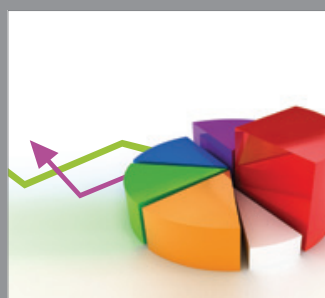

ournal of

Probability and Statistics

Promensencen
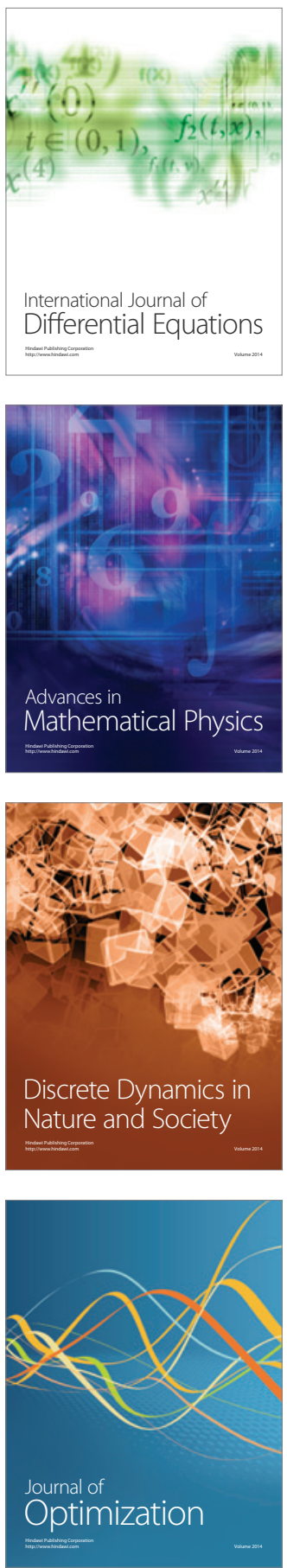